\title{
TRATAMIENTO CON LEVAMISOL DE LA INFECCION POR MANSONELLA OZZARDI
}

\author{
Marcos RESTREPO (1) \& Nubls OCHOA (2)
}

\section{R E S U M E N}

La administración de levamisol a la dosis para adultos, de $150 \mathrm{mg}$ durante 2 a 3 meses, estuvo asociada la disminución y finalmente a la desaparición de microfilarias circulantes de Mansonella ozzardi. No se presentaron reacciones secundarias a la droga y la eosinofilia cireulante, sirvió como indicador de la presencia de microfilarias.

UNITERMOS: Mansonella ozzardi - Tratamiento con levamisol

\section{N T R O D U C C I O N}

La filariasis producida por Mansonella ozzardi es propia de América tropical. Predomina en grupos de población localizados en zonas cálidas y selváticas. En Colombia (Fig. 1) se han encontrado en varias localidades: a) Amazonas colombiano 1-3; b); Vichada, Vaupés, Meta y Casanare 1,47; y en Chocó y Norte de Antioquia 1,8. Por ser una parasitosis de pocas consecuencias clínicas y restringida a regiones selváticas, se estudia muy poco. No se ha descrito un síndrome o enfermedad definida y los pacientes solo manifiestan síntomas inespecífi$\cos$ y muchos individuos infectados son aparentemente asintomáticos.

El tratamiento de la mansonelosis con las drogas empleadas para otras filariasis, no ha sido exitoso. La dietilcarbamazina es efectiva por vía oral para el tratamiento de la filariasis por Wuchereria bancrofti, con ella se consigue la desaparición de las microfilarias circulantes y probablemente la muerte de los parásitos adultos 9-11. Esta droga se ha ensayado en el tratamiento de Mansonella ozzardi, con resultados contradictorios 11,14, aunque la mayoría de los investigadores están de acuerdo en la no eficacia de la droga para esta filariasis.
Con el presente informe queremos evaluar 1a efectividad del levamisol por si solo, en el tratamiento de la mansonelosis. El levamisol es un compuesto imidaźlico que se utilizo inicialmente como antihelmintico intestinal y posteriormente se le encontró que tenía propiedades inmunoreguladoras, aunque en algunos aspectos es una droga controvertida en el cam. po inmunológico ${ }^{15-18}$.

\section{MATERIALES Y METODOS}

Se estudiaron y trataron 4 pacientes, con Mansonella ozzardi. Estos pacientes fueron tratados con levamisol y seguidos parasitológicamente por más de 1 año. A cada uno de ellos se le hizo el diagnóstico por exámenes en fresco con recuento de las microfilarias en $0.02 \mathrm{ml}$ de sangre; también se contaron en el sedimento de la concentración de Knott, utilizando $1 \mathrm{ml}$ de sangre. En sangre circulante se les determinó el porcentaje de eosinófilos durante todo el tiempo del estudio.

\section{INFORME DE LOS CASOS}

Paciente No. 1 (T.G.R.), 44 años, hombre mestizo. Estuvo durante varios años como mi

(1) Laboratorio Departamental de Salud Pública (SSSA). y Corporación para Investigaciones Biologicas (CrB) Medellín Colombia

(2) Laboratorio Departamental de Salud Pública (SSSA) Medellín, Colombia 


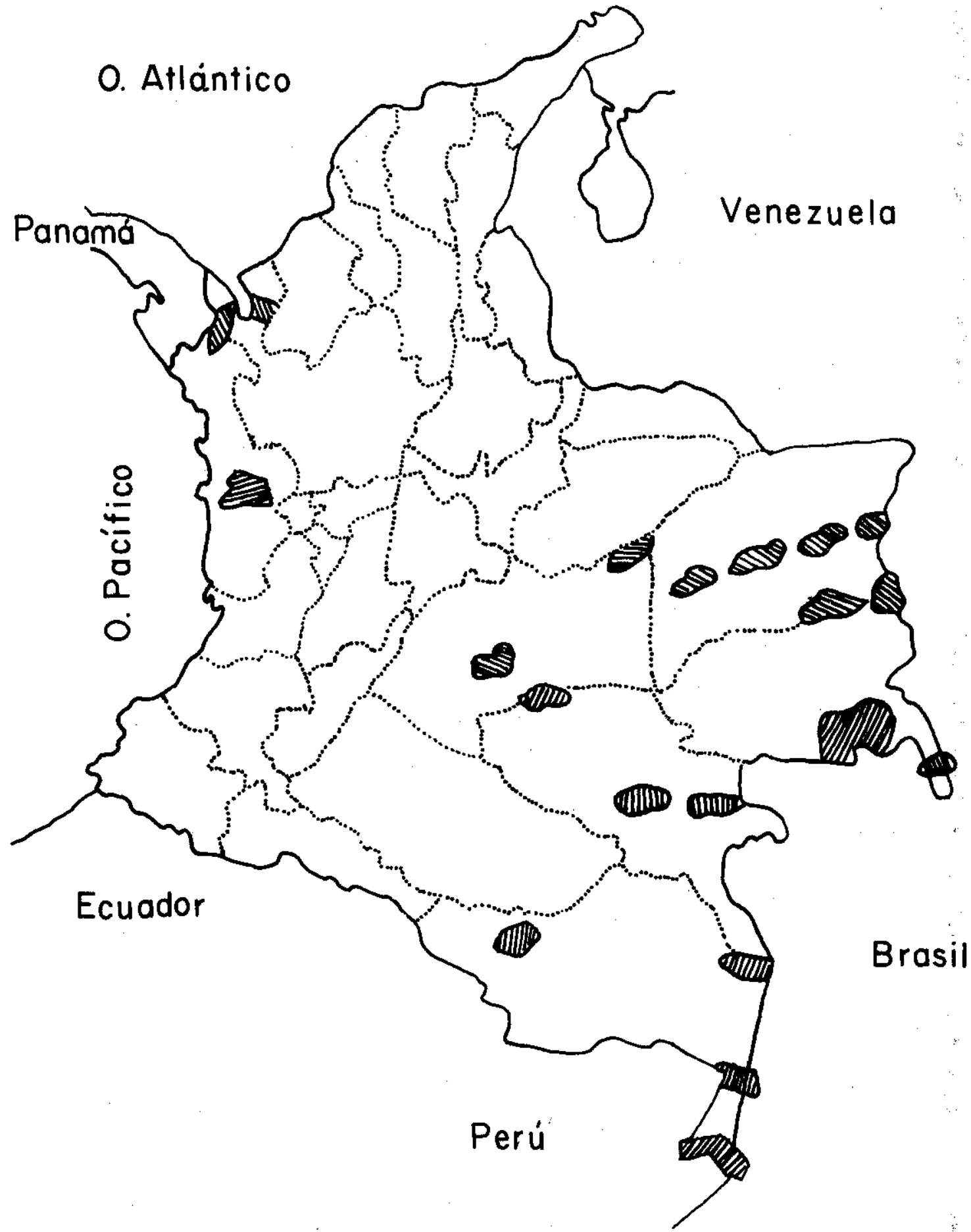

Flg. 1 - Mansonella ozzardf en Colombia

sionero en diferentes sitios del Vaupés, Colom. bia. En 1976 presentó malaria y en las gotas gruesas le observaron microfilarias, que posteriormente fueron clasificadas como Mansonella 
RESTREPO, M. \& OCHOA, N. - Tratamiento con levamisol de la infección por Mansonella orzardi. Rev. Inst. Med. trop. São Paulo, 28:104-110, 1886.

ozzardi. Recibio para esta filariaşis 8 tratamientos con dietilcarbamazina, pero el paciente no pudo precisar la dosis y la duración de cada tratamiento. Tres meses después de la última dosis fue recibido por nosotros. Se le encontraron microfilarias circulantes; a la concentracion de Knott, se contaron 62 microfilarias por ml, (Gráfico 1). Al momento de la consulta el paciente presentaba malestar general, especialmente nocturno, ansiedad, cefalea frecuente de mediana intensidad, dolores abdominales difusos, censación de calor y gripas frecuentes. El examen físico fue normal, incluyendo temperatura y presión arterial. Antes del tratamiento tenía una eosinofilia de $19 \%$. Se inició tratamiento con levamisol, a la dosis de 100 mg/día, los dias lunes, martes y miércoles de cada semana. A los 15 días la microfilaremia bajó notoriamente, pero aún persistía a los 42 días, por este motivo se deciđió administrar la misma dosis pero diariamente. A partir del control hecho a los 72 días, no se observaron microfilarias circulantes, la droga se siguió administrando hasta completar 100 días. Después de suspender el levamisol no se encontró parasitemia. Los eosinófilos, por el contrario, aumentaron inicialmente hasta un $42 \%$ para descender poco a poco. A los 343 días, la eosinofilia se elevó y se detectaron nuevamente las microfilarias, primero a la concentración de Knott y luego al examen directo varios dias después.

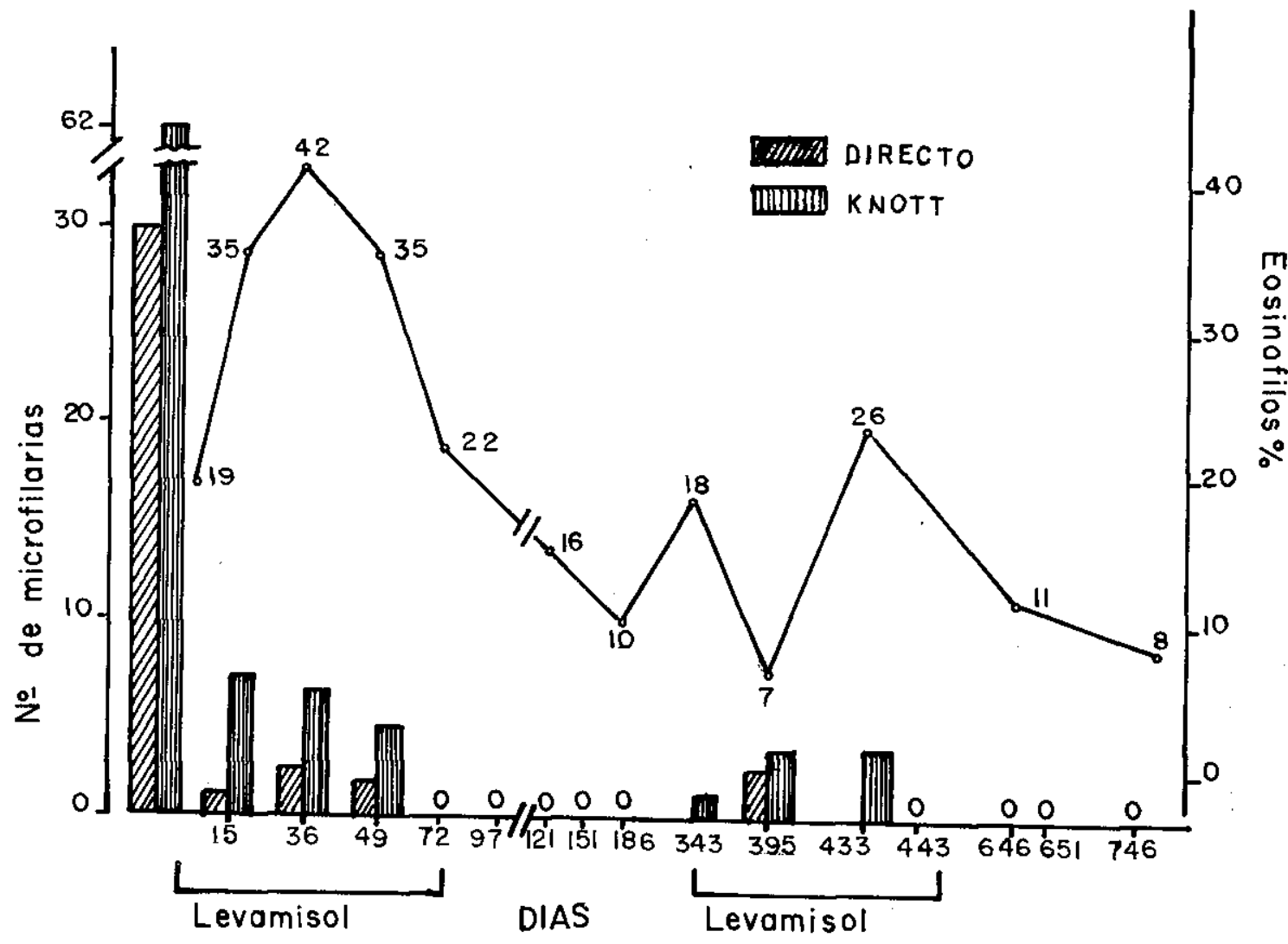

Gráfico No. 1 - Mansonella ozzardi - Levamisol paciente No. 1 (T.G.R.)

Se reestableció el tratamiento con levamisol diariamente a la misma dosis, durante otros 100 días. En el día 443 no se observaron microfilarias y la eosinofilia bajó nuevamente a $11 \%$. Se siguieron los controles en sangre pe- riférica mensualmente hasta 746 días, sin más hallazgos de microfilarias y con eosinofilia de $8 \%$. Durante el tiempo de tratamiento no se encontraron alteraciones hematológicas, excepto la variación en los eosinófilos. El paciente afir- 
mo que se le desaparecieron los síntomas que relató inicialmente, a partir del día 60.

Paciente No. 2 (T.D.R.), 30 años, hombre de raza negra, siempre ha vivido en el Choco; Colombia. Consultó por sintomatologia de paludismo que fué comprobada al laboratorio; además de Plasmodium le observaron microfilarias, por lo cual fue remitido a nosotros para estudio y tratamiento. Al momento de la consulta presentaba malestar general, cefalea frecuente y afirmaba tener brotes de urticaria casi diariamente, que desaparecia espontáneamente. Al examen físico solo se encontró una ligera esplenomegalia, posiblemente como consecuencia de paludismo anteriores. Se encontraron 319 microfilarias de M. ozzardi en $0.02 \mathrm{ml}$ de sangre y 730 a la concentración de Knott. Eosinofilia de $28 \%$. Se inició el levamisol a la dosis diaria de $150 \mathrm{mg}$, durante 50 días (Gráfico 2). Tanto la microfilaremia como la eosinofilia bajaron lentamente a partir del dia 60 , no se detectaron microfilarias circulantes, lo cual se mantuvo hasta 436 días de iniciado el tratamiento. Desde el inicio de la terapia, el paciente afirmó que le habian desaparecido los sintomas.

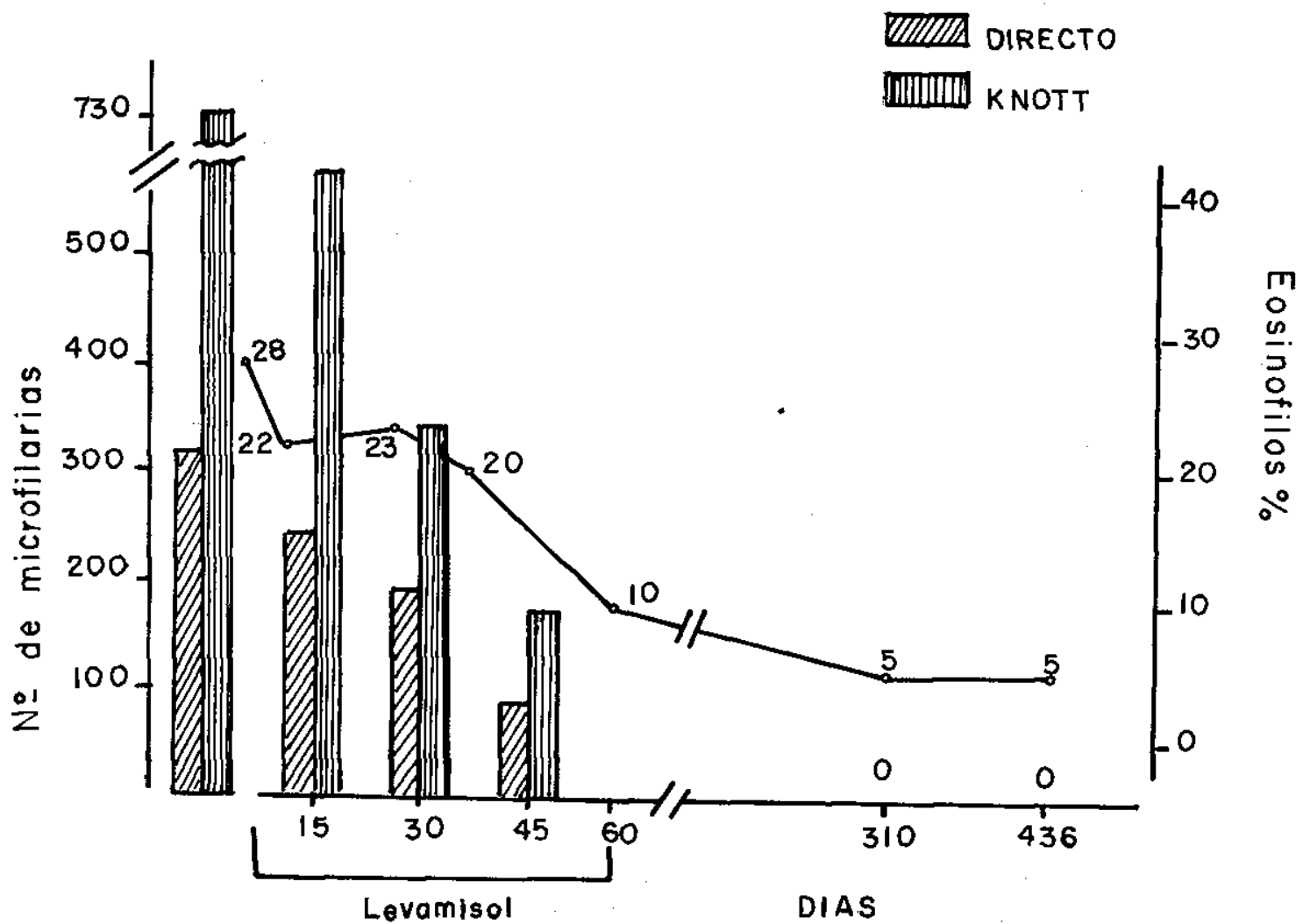

Grafico No, 2 - Mansonella oxzardl - Levamisol paciente No. 2 (T.R.D.)

Paciente No. 3 (J.M.M.) 20 años, mujer de raza negra, nació en Chocó, Colombia y ha vivido en varios sitios de la misma zona. Remitida a Medellín para tratamiento quirúrgico de linfedema crónico desde pie hasta el muslo, que se inició aproximadamente 5 años antes. Además de esta patología, solo manifestaba tener malestar general. Para descartar elefantiasis por filarias, se estudio parasitologicamente durante varios dias, tanto en las horas diurnas como nocturnas. Siempre se encontraron microfilarias cie M. ozzardi, con un promedio de 2 microfilarias en $0.02 \mathrm{ml}$ de sangre y 6 a la concentración de Knott. Recibio levamisol, a la dosis de $150 \mathrm{mgs}$ diarios durante 90 días. Las micro filarias ya no aparecieron a los 30 días. Los eosinófilos disminuyeron también rápidamente. 
RESTREPO, M. \& OCHOA, N. - Tratamiento con levamisol de la infección por Mansanella ozzardi. Rev. Inst. Med. trop. Sāo Panlo, 28:104-110, 1986.

Fue posible seguirla hasta un año sin que reaparecieran las microfilarias.

Paciente No. 4 (A.P.M.), 31 años, hombre de raza negra, procedente del Chocó, Colombia, cerca de Panamá. Fué remitido a nuestra consulta por hallazgo de microfilarias al hacerle un extendido de sangre para un leucograma. Al interrogatorio relata que desde hacía 1 año presentaba astenia, mareos, dolores articulares, cefalea, somnolensia y prurito generalizado. AI estudiarlo encontramos escasas microfilarias de M. ozzardi. Recibió $150 \mathrm{mgs}$ diarios de levamisol durante 30 días. El paciente se negó a continuar la droga porque estaba asintomático y regresó a su localidad; 5 meses después reapareció el paciente porque presentó los mismos síntomas. Se le encontraron escasas microfilarias a la concentración de Knott y se le reinició el mismo tratamiento durante 70 días, el paciente relató que hizo el tratamiento irregularmente, modificando la dosis e interrumpiéndola durante 20.

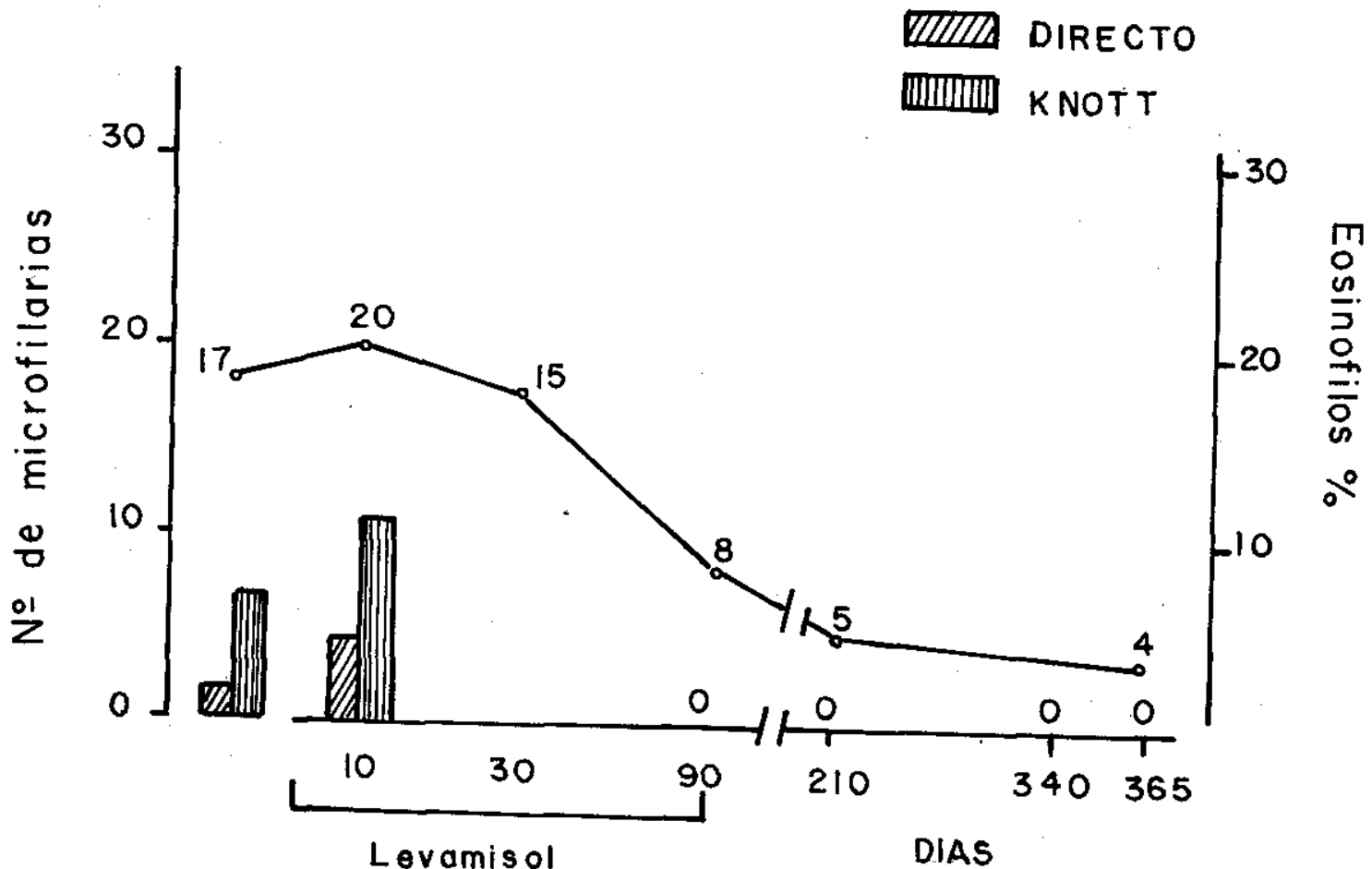

Grafico No. 3 - Mansonella ozzardi - Levamisol pactente No. 3 (J.M.M.)

Finalmente se le dió la última oportunidad de recibir más droga, lo cual hizo durante otros 46 días. A partir del día 316 de iniciar por primera vez el levamisol, no se volvieron a encontrar microfilarias hasta los 350 días, en que se estudio parasitológicamente por última vez, pues nunca regreso a otros controles.

\section{DISCUSION}

PEREZ \& col. en $1980^{19}$ publicaron sus experiencias con el levamisol en el tratamiento de la filariasis por Wuchereria bancrofti, observando una rápida dismunución de las microfi- larias circulantes; el $87.5 \%$ de ellos llegaron a ser negativos y el resto persistieron positivos con microfilaremia baja. MAK \& ZAMAN 30 cbtuvieron experiencia similar, pero la desapa. rición de las microfilarias dependió del número de dias que recibieron la droga. Otros Auto. res ${ }^{21-22}$ informaron efectos potenciadores en su acción contra las filarias del levamisol con dietilcarbamacina para $\mathbf{W}$. bancrofti. También con siguieron efecto potenciador del levamisol con mebendazol para Onchocerca volvulus y Dipe talonema perstans ${ }^{23-25}$.

El presente informe muestra que el leva. misol tiene acción por sí śolo para disminuír 
RESTREPO, M. \& OCHOA, N. - Tratamiento con levamisol de la infección por Mansomella carardi. Rev. Inst. Med. trop. São Paulo, 28:104-110, 1986.

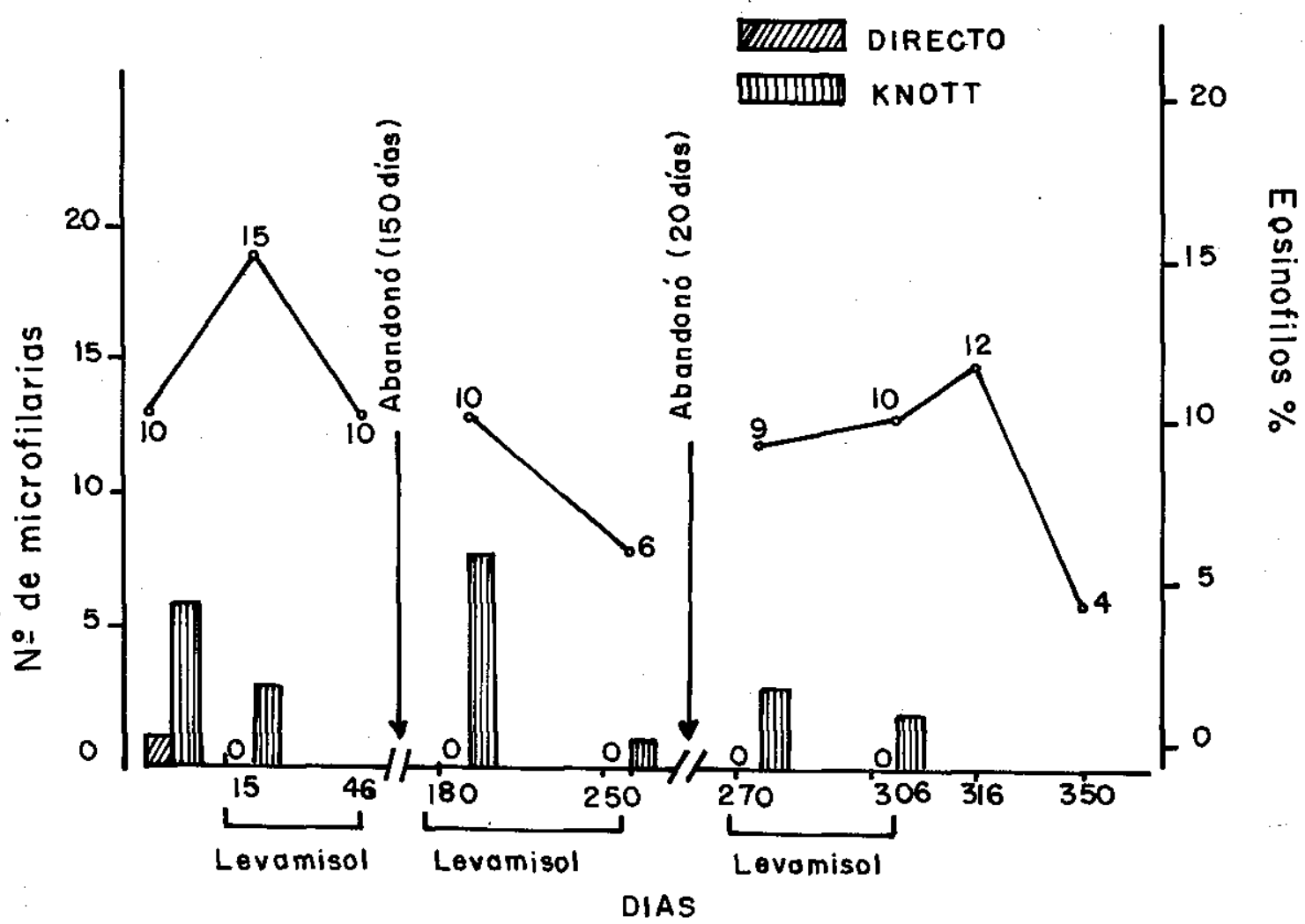

Gráfico No. 4 - Mansonelı ozzardi - Levamisol paciente No. 4 (A.P.M.)

o desaparecer la microfilaremia de Mansonella ozzardi, cuando se administra $2.53 \mathrm{mg} / \mathrm{kg}$ (150 mg para adultos) diariamente durante más de 30 dias. Como se han informado efectos antifilaríasicos del mebendazol para otras filarias distinta a M. ozzardi ${ }^{23-27}$, se sugiere ensayar la asociación de levamisol y mebendazol, para evaluar la posibilidad de acortar el tiempo de tratamiento.

Aunque se han informado reacciones adversas al levamisol, especialmente en tratamientos prolongados ${ }^{10}$, no observamos en nuestros pacientes reacciones secundarias. La determinación de la eosinofilia, sirve para el seguimiento del tratamiento, pues la microfilaremia coincide con la elevación de los eosinófilos circulantes, que al desaparecer las microfilarias, la eosinofilia baja a niveles normales.

\section{SUMMARY}

Mansonella ozzardi infection treated by levamisole.
Administration of levamisole at the adult dosage $(150 \mathrm{mg} /$ day) for period of $2-3$ months, was found to be associated with diminishing numbers and late disappearence of circulating Mansonella ozzardi microfilarias. No adverse reactions to the orug were recorded. The degree of circulating eosinophils served as indicator of the microtilaria presence.

\section{AGRADEGIMIENTOS}

Al Dr. D. Botero, Facultad de Medicina, Universidad de Antioquia por el envio de uso de los pacientes.

\section{REFERENCIAS}

1. ESSLINGER, J. H. \& JIMENEZ, A. - Human In fection with the Flaria Mansonella oxzard in Colom bia. Bull. Tulane med. Fac., 27; 87.91, 1968.

2. RESTREPO, M. - Estudio parasitológlco de una región del Amazonas colombiano. Antioquia méd., 12: 462-484, 1962.

3. KOZEK, W. J.; D'ALESSANDRO, A.; SILVA, J. \& NAVARRETTE, S. N. - Filariosis in Colombia: pre 
RESTREPO, M. \& OCHOA, N. - Tratamiento con levamisol de la infección por Mansonella ozzardi. Rev. Inst. Med. trop. São Paulo, 28:104-110, 1986.

valence of Mansonelia in the teengge and adult population of the Colombian bank of the Amazon, Comisaría del Amazonas, Amer. J, trop. Med. Hyg., 31: 1131. I136, 1982.

4. RESTREPO, M. ; BRAVO, C.; MEJIA, W.; BOTERO, V. M. \& ABAD, H. - Estudio clínico y epidemiológico de una población de los IIanos Orientales, Colombia. Antioquia méd., 15: 283-284, 1965.

5. MARINKELLE, C. \& GERMAN, E. - Mansonellosis in the Comisaria del Vaupes of Colombia. Trop. Geogr. Med., 22: 101-111, 1970.

6. KOZEK, W. J.; PALMA, G.; HENAO, A.; GARCIA, H. \& HOYOS, M. - Filariasis in Colombia: Prevalence and distribution of Mansonella ozzardi and Mansonella (Dipetalonema) perstans infections in the Comisaria del Guainia. Amer. J. trop. Med. Hyg., 32: 379-384, 1983.

7. KOZEK, W. J.; PALMA, G.; VALENCIA, W.; MONTALVO, C. \& SPAIN, J. - Filariasis in Colombia: Prevalence of Mansonella ozzardi in the departamento del Meta, Intendencia del Casanare and Comisaria del Vichada. Amer. J. trop. Hyg., 33: 70-72, 1984.

8. RESTREPO, M. ; LATORRE, R. \& BOTERO, D. - Presentación de un caso de mansonelosis. Antioquia méd., 12: 233-242, 1862.

9. MANSON-BAHR, P. E. C. \& WIJERS, D. J. B. The effect of a small dose of diethylcarbamazine on the circulation in the blood of microfilariae of $\mathbf{w}$. ban. crofti. Trans. roy. Soc. trop. Med. Hyg., 66: 18, 1972.

10. RUSSEL, S.; SUNDARAM, R. M.; CHANDRASEKHARAN, A. \& RAO, C. K. - Provocative effect of day. time administration of diethycarbamazine on microfilaraemia of Wuchereria bancrofti and Brugia malayi. J. Communicat. Disor., \%: 59-64, 1975.

11. PARTONO, F.; PURNOMO; OEMIJATI, S. \& SOEWAR. TA, A. - The long term effects of repeated diethylcarbamazine administration with special reference to $\mathrm{mi}$ crofilaremia and elephantiasis. Acta trop. (Basel), 38: 217-225, 1981.

12. MAZZOTI, L. - Resultados negativos de la administración del "Hetrazán" en dos pacientes infectados con Mansonella ozzardi. Medicina (Méx.), 28: 317-318, 1948.

13. MONTESTRUC, E.; BLANCHE, R. \& LABORDE, R. Action du 1-diéthylcarbamyl 4-methylpiperazine sur Filaria ozzardi. Bull. Soc. Path. exot., 43: 275-278, 1950.

14. BARTHOLOMEW, C. F.; NATHAN, M. B. \& TIKA. SINGH, E. S. - The failure of diethylcarbamazine in the treatment of Mansonella ozzardi infections. Trans. roy. Soc. trop. Med. Hyg., 72: 423-424, 1978.

15. THIENPONT, D.; BRUGMANS, J.; ABADI, K. \& TANAMAL, S. - Evaluation of tetramisol in the treatment of nematode infection in man. Amer. J. trop. Med. Hyg., 18: 520-525, 1969.

16. SYMOENS, J. et al. - Levamisole. Pharmacological and Biochemical Properties of Drug Sustances. Am. Pharmaceut. Ass., 1979. Vol. 2.

17. SYMENS, J.; VEYS, E.; MIELANTS, M. \& PINALS, R - Adverse reactions to levamisole. Cancer Treat. Rep. 62: 1721-1730, 1978.

18. GOLDSTEIN, L. - Mode of action of Levamisole. J. Rheum., 5 (Suppl. 4): 143-148, 1978.

19. PEREZ, J.; I.ASTRE, M.; LAZO, O. \& MONTAIVO, G. - Uso del Levamisol en la fllariasis por $\mathbf{W}$. bancroft. Rev. cuba. Med. trop., 32: 185-190, 1980.

20. MAK, J. W. \& ZAMAN, V. - Levamisole hydrochloride in Brugia malayi and Buchereria bancrofti Infection. H1th. Policies Develop. Countries: Roy. Soc. Med. Int $x_{x}$ Congress - Symposium Series No. 24. London, Academic Press, 1979.

21. LOUIS, F. J. \& LAIGRET, J. - Essal de l'association diethylcarbamazine (Notézine) Levamisole (Solaskil) dans la lutte contre la filariose a Wuchereria bancrotti, var. pacifica, en Polynésie Francaise. Bull. Soc. Path. exot., 72: 471-481, 1979.

22. TEMU, S. E. \& MCMAHON, J. E. - Chemotherapy with spaced doses of dethylcarbamazine preceded by levamisole in Bancroftian Fllariasis. Trans, roy. Soc. trop. Med. Hyg., 75: 835-837, 1981.

23. MAERTENS, K. \& WERY, M. - Effect of mebendazole and levamisole on Onchocerca volvulus and Dipetalonema perstans. Trans. roy. Soc. trop. Mcd. Hyg., 69: $359-360,1975$.

24. BERNBERG, H. C.; ClaRKE, V. de V. \& GELFAND, M. - The combined treatment with levamisole and me. bendazole for a perstans-like filarial infection in Rho. desia. Trans. roy. Soc. trop. Med. Hyg., 73: 233-234, 1979.

25. BOSSCHE, H. D. - Antiflarial activity of Levamisole - Mebendazole-Flubendazole. Hith. Policies Develop. Countries: Roy. Soc. Med. Int. Congress-Symposium Series No. 24. London, Academic Press, 1880.

26. WAHLGREN, M. - The successful treatment of Dipetalonema perstans with mebendazole. Ann. trop. Med. Parasit., 76: 557-579, 1982.

27. WAHLGREN, M. \& FROLOV, I. - Treatment of Dipetalonema perstans infections with mebendazole. Trans. roy. Soc. trop. Med. Hyg., 77: 422-423, 1983.

Recebido para publicaçăo em $28 / 5 / 1985$. 